Homology, Homotopy and Applications, vol.19(1), 2017, pp.89-110

\title{
REMARKS ABOUT $\Delta$-COMPLEXES AND APPLICATIONS
}

\author{
MICHAEL PORS, SOUMEN SARKAR AND PETER ZVENGROWSKI
}

(communicated by Donald M. Davis)

\begin{abstract}
We first consider some generalities regarding $\Delta$-complexes, in particular, we give a brief history of the category of $\Delta$-complexes, and its relation to the category of semi-simplicial complexes introduced in 1950 by Eilenberg and Zilber. A natural construction of $\Delta$-complexes arising from a group action on a simplicial complex is next considered. Finally, an application of this construction to obtain an elementary explicit computation of the cohomology ring $H^{*}\left(\mathbb{R} P^{n} ; \mathbb{Z}_{2}\right)$, based on a $\Delta$-complex structure, is given.
\end{abstract}

\section{Introduction}

The idea and terminology of $\Delta$-complexes seems to originate in about 2002 in the book of Hatcher [11], indeed it is introduced in Section 2.1, and can now be found in a few undergraduate texts such as $[\mathbf{2}]$. Note that the version of $\Delta$-complexes given in the first edition of [11] was somewhat incomplete, and we will be following the improved version given in the subsequent editions. An earlier version of an equivalent idea, due to Eilenberg and Zilber [7] in 1950, is mentioned by Hatcher. In the Eilenberg-Zilber paper these objects are called "semi-simplicial complexes," which can be confusing since this term has later been used in a different context. To avoid confusion with subsequent usage we shall call the "semi-simplicial complexes" introduced in [7] "EZcomplexes" (face maps only). The "complete semi-simplicial complex" introduced in [7] will be called "simplicial sets" (face maps and degeneracy maps), in agreement with the terminology of texts such as $[\mathbf{1 0}, \mathbf{1 7}, \mathbf{2 2}]$.

In the second section of this note we give a brief historical sketch of this subject and the related idea of geometric realization. In the third section we recall (cf. [11]) the definition of the category of $\Delta$-complexes, denoted by $\Delta$-Comp. The equivalence of this category with the category of EZ-complexes, as suggested in Section 2.1 of [11], is demonstrated. A corollary of this is that the homology functor on $\boldsymbol{\Delta}$-Comp is indeed a homology theory satisfying the Eilenberg-Steenrod axioms, and related constructions such as cup product and Steenrod squares (in cohomology theory), and local coefficients, can be carried out on the category $\boldsymbol{\Delta}$-Comp.

The second author was supported by PIMS, University of Regina and University of Calgary. The third named author was supported during this work by a Discovery Grant from the Natural Sciences and Engineering Research Council of Canada.

Received February 11, 2016, revised April 13, 2016; published on March 22, 2017.

2010 Mathematics Subject Classification: Primary 55N35, Secondary 57Q91.

Key words and phrases: $\Delta$-complex, EZ-complex, group action.

Article available at http://dx.doi.org/10.4310/HHA.2017.v19.n1.a5

Copyright (C) 2017, International Press. Permission to copy for private use granted. 
The fourth section considers a construction of $\Delta$-complexes that frequently occurs in practice, namely via the action of a finite group on a $\Delta$-complex, in particular, on a simplicial complex. The final section then gives an application of this construction to obtaining a $\Delta$-complex structure for $\mathbb{R} P^{n}$ and using this to give an elementary explicit computation of the cohomology ring $H^{*}\left(\mathbb{R} P^{n} ; \mathbb{Z}_{2}\right)$.

\section{A brief history}

The ideas behind the categories we are studying originated in the 1940s with Eilenberg's work on singular homology theory, and were first given formal expression in the 1950 paper [7] of Eilenberg and Zilber. We remind the reader that the terms "semisimplicial complex, complete semi-simplicial complex" in [7] correspond respectively to "EZ-complex, simplicial set" in our terminology. They are respectively denoted by EZ-Comp, Simp. The simplicial sets have received the lion's share of the attention since 1950, probably because of their advantage in dealing with products of spaces and their suitability for abstract homotopy theory. An excellent brief history of the simplicial sets can be found in Weibel's memoir [23], and there are also books dealing with this subject such as $[\mathbf{1 7}]$ by May, [10] by Goerss and Jardine, as well as chapters in other books such as $[\mathbf{2 2}]$ by Selick and various seminar or lecture notes such as $[\mathbf{4}]$ by Curtis.

We therefore concentrate here on the much less extensive history of the EZcomplexes. The singular complex of a topological space gives a functor from spaces to EZ-complexes (and to simplicial sets). Already in 1950 Giever [8] defined an adjoint to this functor, which we now call a geometric realization. In $1951 \mathrm{Hu}[\mathbf{1 3}]$ gave a more general version of this geometric realization, which would, in fact, work for any EZ-complex and is the same as Giever's for the singular complex of a space. The geometric realization of a simplicial set was given in 1957 by Milnor [19], who emphasizes right at the start of his paper that "This construction will be different from that of Giever and $\mathrm{Hu}$ in that the degeneracy operations of $K$ are used." As the titles of the papers by Giever and $\mathrm{Hu}$ indicate, their primary aims were in different directions and the geometric realizations/EZ-complexes were used as a convenient tool.

The next work that deals with EZ-complexes is that of Rourke and Sanderson [21] in 1971. In this paper EZ-complexes are called $\Delta$-sets, while simplicial sets are called semi-simplicial complexes and abbreviated to css-sets. In fact this paper proves that there is an equivalence of these two categories, and then goes on to discussing the Kan condition and developing homotopy theory. Curiously, although Lemma 1.2 in this paper quotes a result of Eilenberg-Zilber, [7] is not mentioned in the bibliography.

It seems that the next appearance of EZ-complexes is not until Hatcher's book in 2002 [11], together with the idea of $\Delta$-complexes, as already mentioned in the Introduction. Two recent papers dealing with EZ-complexes are $[\mathbf{9}, \mathbf{1 8}]$, where they are called semi-simplicial sets.

\section{Generalities about $\Delta$-complexes}

The objective of this section is to recall the category of $\Delta$-complexes, in the sense of Hatcher [11], the category of EZ-complexes following Eilenberg-Zilber [7], and to 
show that these categories are equivalent (cf. MacLane [16, Ch. IV, Theorem 4.1], for the notion of equivalence of categories). In the case of $\Delta$-complexes we give a categorical interpretation as the colimit of a suitable diagram. In the case of EZcomplexes we also give a categorical interpretation as a contravariant functor (cf. Propositions 3.12 and 3.14). Both these interpretations will be convenient for proving the main theorem of this section, Theorem 3.21.

First we fix some notations and terminology. Much of this is standard (cf. [16]), however conventions vary. So we fix them here. Throughout this section a diagram $D$ in a category $\mathbb{X}$ is itself a category that consists of, for some set $I$, an $I$-indexed set

$$
\operatorname{Obj}(D)=\left\{X_{i}: i \in I, X_{i} \in \operatorname{Obj}(\mathbb{X})\right\}
$$

together with, for each $i, j \in I$, a (possibly empty) subset of morphisms

$$
D(i, j) \subseteq \mathbb{X}\left(X_{i}, X_{j}\right)=\operatorname{hom}_{\mathbb{X}}\left(X_{i}, X_{j}\right)
$$

such that, for any $i, j, k \in I$, any $f \in D(i, j)$, and any $g \in D(j, k)$, the composite $g \circ$ $f \in D(i, k)$, and $1_{X_{i}} \in D(i, i)$. Given this formalization a diagram is said to commute if for all $i, j \in I$ the set $D(i, j)$ contains at most one element.

With the diagram $D$ as above, a cocone from the base $D$ to the vertex $X$ (or cocone from $D$ into $X)$ is a pair $\left(X,\left\{\left(X_{i}, \xi_{i}\right)\right\}\right)$ where $X \in \operatorname{Obj}(\mathbb{X})$ and $\left\{\left(X_{i}, \xi_{i}\right)\right\}=$ $\left\{\left(X_{i}, \xi_{i}\right): i \in I\right\}$ is an $I$-tuple of pairs $\left(X_{i}, \xi_{i}\right)$ with each $X_{i} \in \operatorname{Obj}(\mathbb{X})$ and each $\xi_{i}: X_{i} \rightarrow X$ a morphism in $\operatorname{hom}_{\mathbb{X}}\left(X_{i}, X\right)$ such that, for all $f \in D(i, j)$, the diagram

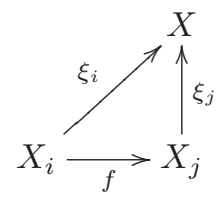

commutes. A colimit of a diagram $D$ is then a cocone $\left(X_{D},\left\{\left(X_{i}, \phi_{i}\right)\right\}\right)$ such that, for any other cocone $\left(X,\left\{\left(X_{i}, \xi_{i}\right)\right\}\right)$, there is a unique morphism $\xi: X_{D} \rightarrow X$ such that, for all $i \in I$ the diagram

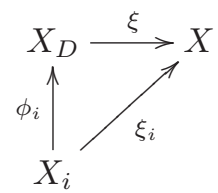

commutes. One also writes $X_{D}=\operatorname{colim} D$. Since colimits are unique up to unique isomorphism (cf. [16, Ch. III, §4]) we will often refer to the colimit of a diagram $D$. The dual notion of a cone is used in $[\mathbf{1 6}]$ to define limits.

Definition 3.1. (i) For any $n, m \in \mathbb{N}$ let $R=\left\{v_{0}<\cdots<v_{n}\right\}$ be an ordered set of $n+1$ elements of $\mathbb{R}^{m}$ such that the set $\left\{v_{i}-v_{0}: 1 \leqslant i \leqslant n\right\}$ is linearly independent. The resulting convex-hull

$$
\Delta_{R}^{n}:=\left\{\sum_{i=0}^{n} t_{i} v_{i}: t_{0}, \ldots, t_{n} \geqslant 0 \text { and } \sum_{i=0}^{n} t_{i}=1\right\}
$$

is called the ordered $n$-simplex generated by $R$ in $\mathbb{R}^{m}$, and will also be written $\left\langle v_{0}, \ldots, v_{n}\right\rangle$. We call $v_{i}$ the $i$-th vertex of $\Delta_{R}^{n}$. Given ordered $n$-simplexes $\Delta_{R}^{n}$ and 
$\Delta_{S}^{n}$ there is a canonical affine homeomorphism

$$
\Delta_{R}^{n} \rightarrow \Delta_{S}^{n} \quad \text { given by } \quad \sum_{i=0}^{n} t_{i} u_{i} \longmapsto \sum_{i=0}^{n} t_{i} v_{i} .
$$

The standard $n$-simplex $\Delta^{n}$ in $\mathbb{R}^{n+1}$ is obtained by taking $v_{i}=e_{i+1}$ for $0 \leqslant i \leqslant n$.

(ii) Given an $n$-simplex $\Delta_{R}^{n}$ and a natural number $k \leqslant n$, a $k$-face of $\Delta_{R}^{n}$ is a $k$ simplex generated by $k+1$ vertices of $\Delta_{R}^{n}$ with the induced ordering. Notice that there are $\left(\begin{array}{l}n+1 \\ k+1\end{array}\right) k$-faces of $\Delta_{R}^{n}$. If $K$ is a $k$-face of $\Delta_{R}^{n}$ then the $k$-face inclusion of $\Delta^{k}$ into $\Delta_{R}^{n}$ associated with $K$ is the composition $\iota_{K}^{n}$ of the canonical affine homeomorphism from $\Delta^{k}$ to $K$ followed by subset inclusion

$$
\Delta^{k} \stackrel{\cong}{\longrightarrow} \longrightarrow \Delta_{R}^{n}
$$

In particular, if $K$ is an $(n-1)$-face of the standard $n$-simplex $\Delta^{n}$, let $k$ be the index of the unique vertex not contained in $K$. One denotes the corresponding face inclusion by

$$
\iota_{k}^{n}: \Delta^{n-1} \rightarrow \Delta^{n} .
$$

(iii) Given an $n$-simplex $\Delta^{n}$, let $\partial \Delta^{n}$, called the boundary of $\Delta^{n}$, denote the union of the $(n-1)$-faces of $\Delta^{n}$. Let $\stackrel{\circ}{\Delta}^{n}:=\Delta^{n} \backslash \partial \Delta^{n}$ and call it the interior of $\Delta^{n}$. Notice that $\partial \Delta^{0}=\emptyset$ and $\stackrel{\circ}{\Delta}^{0}=\Delta^{0}$. This may seem odd, but it simplifies notation later.

This next definition is taken from Section 2.1 of [11] with the only modifications being a slight categorification of the language. See the same section of [11] for several examples (and non-examples). By a "map" we always mean a continuous function.

Definition 3.2. A $\Delta$-complex is a pair $\mathcal{X}=(X, \Sigma)$ consisting of a topological space $X$ and a collection of maps

$$
\Sigma=\left\{\sigma_{\alpha}: \Delta^{n_{\alpha}} \rightarrow X: \alpha \in \mathcal{A}, n_{\alpha} \geqslant 0\right\}
$$

satisfying the following conditions.

A1. For each $\sigma_{\alpha} \in \Sigma$ the composite $\stackrel{\circ}{\Delta}^{n_{\alpha}} \hookrightarrow \Delta^{n_{\alpha}} \stackrel{\sigma_{\alpha}}{\longrightarrow} X$ is injective. Call this composite $\stackrel{\circ}{\sigma}_{\alpha}$. Definition 3.1 (iii) implies that whenever $n_{\alpha}=0$, we get that $\stackrel{\circ}{\sigma}_{\alpha}=\sigma_{\alpha}$.

A2. Each $x \in X$ is in the image of exactly one such $\stackrel{\circ}{\sigma}_{\alpha}$.

A3. For each $\sigma_{\alpha}: \Delta^{n_{\alpha}} \rightarrow X$ in $\Sigma$, each $k<n_{\alpha}$, and every face inclusion $\Delta^{k} \hookrightarrow \Delta^{n_{\alpha}}$, the composite $\Delta^{k} \hookrightarrow \Delta^{n_{\alpha}} \stackrel{\sigma_{\alpha}}{\longrightarrow} X$ is one of the maps in $\Sigma$.

A4. A set $A \subseteq X$ is open if and only if $\sigma_{\alpha}^{-1}(A)$ is open in $\Delta^{n_{\alpha}}$ for each $\sigma_{\alpha} \in \Sigma$.

We write $\Sigma_{n}=\left\{\sigma_{\alpha}: n_{\alpha}=n\right\} \subseteq \Sigma$, and call each $\sigma_{\alpha} \in \Sigma_{n}$ an $n$-simplex of $\mathcal{X}$.

Definition 3.3. A morphism $\mathcal{X}=(X, \Sigma) \rightarrow \mathcal{Y}=\left(Y, \Sigma^{\prime}\right)$ of $\Delta$-complexes is a map $f: X \rightarrow Y$ such that, for each $\sigma \in \Sigma$ we get that the composite

$$
\Delta^{n_{\alpha}} \stackrel{\sigma_{\alpha}}{\longrightarrow} X \stackrel{f}{\longrightarrow} Y
$$

is an element of $\Sigma^{\prime}$. By a slight abuse of notation, we also write $f: \mathcal{X} \rightarrow \mathcal{Y}$. 
Example 3.4. If $X$ is a $\Delta$-complex with associated maps $\Sigma$, then the identity map $1_{X}: X \rightarrow X$ clearly satisfies $1_{X} \circ \sigma \in \Sigma$ for all $\sigma \in \Sigma$, hence $1_{X}$ is a morphism of $\Delta$-complexes.

Proposition 3.5. There is a category, $\Delta$-Comp, whose

- objects are $\Delta$-complexes,

- morphisms are morphisms of $\Delta$-complexes,

- identity morphisms are the identity maps of Example 3.4,

- composition is given by the usual composition of functions.

Proof. This is routine to check.

Example 3.6. Any finite simplicial complex (see Definition 4.2) is a $\Delta$-complex, cf. [2, Section 6.1$]$. To obtain a $\Delta$-complex structure one may use a partial order $\preceq$ for the vertices such that the vertices of any simplex are simply ordered under $\preceq$, for example, any total order of the vertices of the simplicial complex will suffice. Note that different partial orders may give rise to different $\Delta$-complex structures. In particular, we may regard $\Delta^{n}$ as a $\Delta$-complex (with $e_{1}<\ldots<e_{n+1}$ ), which we denote simply as $\Delta^{n}$ (or $\left.\left(\Delta^{n}, \Sigma_{n}\right)\right)$, and any face inclusion $\Delta^{k} \stackrel{\iota}{\longrightarrow} \Delta^{n}$ is a morphism of $\Delta$-complexes.

The next lemma identifies a $\Delta$-complex as a suitable colimit, and will be useful towards proving the main Theorem 3.21. A similar result, for simplicial sets, can be found in Section 1.2 of [10].

Lemma 3.7. Let $\mathcal{X}=(X, \Sigma)$ be a $\Delta$-complex, and let $D=D_{\mathcal{X}}$ be the diagram in the category $\Delta$-Comp with one copy of $\Delta^{n_{\alpha}}$ for each $\sigma_{\alpha} \in \Sigma$, denoted $\left(\Delta^{n_{\alpha}}, \sigma_{\alpha}\right)$, and a face inclusion $\iota: \Delta^{n_{\alpha}} \rightarrow \Delta^{n_{\beta}}$ if $\sigma_{\alpha}$ factors as

$$
\sigma_{\beta} \circ \iota=\sigma_{\alpha}
$$

Then $\mathcal{X}$ is the colimit of $D$.

Proof. Let $\iota: \Delta^{n_{\alpha}} \rightarrow \Delta^{n_{\beta}}$ be a morphism of $D$, then by construction we know that

$$
\sigma_{\beta} \circ \iota=\sigma_{\alpha} .
$$

Thus $(X, \Sigma)$ is a cocone for $D$. Let $\left(Y, \Sigma^{\prime}\right)$ be another cocone for $D$, where $\Sigma^{\prime}=$ $\left\{\phi_{\alpha}: \sigma_{\alpha} \in \Sigma\right\}, \phi_{\alpha}: \Delta^{n_{\alpha}} \rightarrow Y$. We need to show that there is a unique map $\phi: X \rightarrow Y$ such that $\phi \sigma_{\alpha}=\phi_{\alpha}$ for all $\sigma_{\alpha} \in \Sigma$.

By A2, for each point $x \in X$ there is a unique $\sigma_{\alpha(x)} \in \Sigma$ and a unique $p_{x} \in \stackrel{\circ}{\Delta}^{n_{\alpha(x)}}$ such that

$$
x=\stackrel{\circ}{\sigma}_{\alpha(x)}\left(p_{x}\right) .
$$

Define $\phi: X \rightarrow Y$ by

$$
\phi(x):=\phi_{\alpha(x)}\left(p_{x}\right)
$$

We now show $\phi$ is a morphism of cocones for $D$. Indeed, for any $p \in \Delta^{n_{\alpha}}$, if $p \in \stackrel{\circ}{\Delta}^{n_{\alpha}}$ then $\left(\phi \sigma_{\alpha}\right)(p)=\phi_{\alpha}(p)$ from the above definition of $\phi$, taking $\alpha(x)=\alpha$ and $x=\sigma_{\alpha}(p)$. 
Otherwise let $\Delta^{n_{\beta}}$ be the face of $\Delta^{n_{\alpha}}$ such that $p \in \stackrel{\circ}{\Delta}^{n_{\beta}}$ and let

$$
\iota: \Delta^{n_{\beta}} \rightarrow \Delta^{n_{\alpha}}
$$

be the associated face inclusion. By hypothesis $p=\iota\left(p^{\prime}\right)$ for some $p^{\prime} \in \Delta^{n_{\beta}}$, and compute

$$
\begin{aligned}
\left(\phi \sigma_{\alpha}\right)(p) & =\phi\left(\sigma_{\alpha}(p)\right) \\
& =\phi_{\beta}\left(p^{\prime}\right) \\
& =\left(\phi_{\alpha} \iota\right)\left(p^{\prime}\right) \\
& =\phi_{\alpha}\left(\iota\left(p^{\prime}\right)\right) \\
& =\phi_{\alpha}(p) .
\end{aligned}
$$

(by our choice of $\beta$ above and our definition of $\phi$ )

(since $\phi$ is a cocone)

Hence $\phi \circ \sigma_{\alpha}=\phi_{\alpha}$ for each $\sigma_{\alpha} \in \Sigma$.

Next we show that $\phi$ is continuous. Let $A \subseteq Y$ be open and let $B:=\phi^{-1}(A)$. By definition of the topology on $X$ we have that $B$ is open if and only if $\sigma_{\alpha}^{-1}(B)$ is open for all $\sigma_{\alpha} \in \Sigma$, but

$$
\sigma_{\alpha}^{-1}(B)=\sigma_{\alpha}^{-1}\left(\phi^{-1}(A)\right)=\left(\phi \sigma_{\alpha}\right)^{-1}(A)=\phi_{\alpha}^{-1}(A)
$$

and $\phi_{\alpha}^{-1}(A)$ is open since $\phi_{\alpha}$ is continuous by assumption. We conclude that $B$ is open, and hence that $\phi$ is continuous.

Finally, to see that $\phi$ is unique, let $\xi: X \rightarrow Y$ likewise satisfy $\xi \circ \sigma_{\alpha}=\phi_{\alpha}$ for each $\sigma_{\alpha} \in \Sigma$. Now for each $x \in X$ there is a $\sigma_{\beta} \in \Sigma$ such that $x \in \operatorname{Im} \sigma_{\beta}$, say $x=\sigma_{\beta}(p)$. In particular, we get that

$$
\phi(x)=\phi\left(\sigma_{\beta}(p)\right)=\left(\phi \sigma_{\beta}\right)(p)=\phi_{\beta}(p)=\left(\xi \sigma_{\beta}\right)(p)=\xi\left(\sigma_{\beta}(p)\right)=\xi(x) .
$$

Since $x$ was arbitrary we conclude that $\phi=\xi$. Thus $\phi$ is the unique map with this property.

It follows that colim $D$ exists in the category $\boldsymbol{\Delta}$-Comp and equals to $\mathcal{X}$.

Remark 3.8. If $\mathcal{X}$ and $\mathcal{Y}$ are $\Delta$-complexes, then a morphism $f: \mathcal{X} \rightarrow \mathcal{Y}$ gives rise to a functor $F: D_{\mathcal{X}} \rightarrow D_{\mathcal{Y}}$, where $F\left(\Delta^{n_{\alpha}}, \sigma_{\alpha}\right)=\left(\Delta^{n_{\alpha}}, f \circ \sigma_{\alpha}\right)$, such that

$$
f=\operatorname{colim} F: \mathcal{X}=\operatorname{colim} D_{\mathcal{X}} \rightarrow \operatorname{colim} D_{\mathcal{Y}}=\mathcal{Y} \text {. }
$$

Conversely, any functor $F: D_{\mathcal{X}} \rightarrow D_{\mathcal{Y}}$ arises in this way from a morphism $f: \mathcal{X} \rightarrow \mathcal{Y}$ of $\Delta$-complexes where, for $x \in X$, say $\stackrel{\circ}{\sigma}_{\alpha}(\xi)=x \in \operatorname{Im} \stackrel{\circ}{\sigma}_{\alpha}$ (uniquely) and $F\left(\Delta^{n_{\alpha}}, \sigma_{\alpha}\right)=$ $\left(\Delta^{n_{\alpha}}, \tau_{\alpha}\right)$, we set $f(x)=\tau_{\alpha}(\xi) \in Y$.

Now we define the category of EZ-complexes, EZ-Comp. This definition is from [7] with minor changes in terminology and notation. In particular, we use the term "EZcomplex" in place of "semi-simplicial complex", and we use an $\mathbb{N}$-indexed collection of sets $K_{n}$ in place of a set $K$ together with a function $K \rightarrow \mathbb{N}$ (here $0 \in \mathbb{N}$ ).

Definition 3.9 (Eilenberg-Zilber [7]). An EZ-complex, $K$, is an $\mathbb{N}$-indexed collection of sets $K_{n}$ together with a collection of functions

$$
\partial_{i}^{n}: K_{n} \rightarrow K_{n-1}
$$

for all $0<n \in \mathbb{N}$ and all integers $0 \leqslant i \leqslant n$. These functions must satisfy the identity

$$
\partial_{i}^{n-1} \partial_{j}^{n}=\partial_{j-1}^{n-1} \partial_{i}^{n}
$$

for all $n \in \mathbb{N}$ and all $i<j$. Call the elements of $K_{n}$ the $n$-simplexes of $K$. For any 
$n$-simplex $\sigma$ call the $(n-1)$-simplex $\partial_{i}^{n}(\sigma)$ the $i$ th face of $\sigma$.

Note that for the above Definition 3.9, May [17] uses the notation $\partial_{i}$, Selick $[\mathbf{2 2}]$ uses $d_{i}$ and $\sigma^{(i)}$ is used in [7]. Our aim now is to define a category $\widetilde{\Delta}$ such that any EZ-complex may be described as a contravariant functor from $\widetilde{\Delta}$ to Set. The same idea occurs in [21], and for simplicial sets can be found in $[\mathbf{1 7}$, p. 4], [22, p. 91] and partly in $[7, \S 8]$.

Definition 3.10. Let $\widetilde{\Delta}$ be the category whose

- objects are the ordered sets $[n]=\{0<1<2<\cdots<n\}$ for each $n \in \mathbb{N}$,

- morphisms are the injective order-preserving functions,

- identities are the identity functions,

- composition is the usual composition of functions.

For every $n \in \mathbb{N}$ and every integer $0 \leqslant i \leqslant n+1$ define $\delta_{i}^{n}:[n] \rightarrow[n+1]$ by

$$
\delta_{i}^{n}(k)= \begin{cases}k, & k<i, \\ k+1, & k \geqslant i .\end{cases}
$$

These functions are all clearly order-preserving and injective, hence they are morphisms of $\widetilde{\Delta}$.

Lemma 3.11. For any $n \in \mathbb{N}$ the equation

$$
\delta_{j}^{n+1} \circ \delta_{i}^{n}=\delta_{i}^{n+1} \circ \delta_{j-1}^{n}
$$

holds for every $0 \leqslant i<j \leqslant n+2$.

The proof is a routine computation. The next result follows immediately from contravariance and Lemma 3.11.

Proposition 3.12. Given a contravariant functor $L: \widetilde{\Delta} \rightarrow$ Set, the sets $K_{n}:=L([n])$ together with the morphisms $\partial_{j}^{n}:=L\left(\delta_{j}^{n-1}\right)$ for all $n \in \mathbb{N}$ and all $0 \leqslant j \leqslant n$ form an EZ-complex $K:=K_{L}$.

Lemma 3.13. Every non-identity morphism $\mu:[n] \rightarrow[n+m]$ in $\widetilde{\Delta}$ can be uniquely written as a composite

$$
\mu=\delta_{k_{m-1}}^{n+m-1} \circ \cdots \circ \delta_{k_{1}}^{n+1} \circ \delta_{k_{0}}^{n} \quad \text { with } \quad k_{0}<k_{1}<\cdots<k_{m-1} .
$$

Proof. This can be found in (8.3) of Section 8 of [7] (ignoring the degeneracy maps).

Proposition 3.14. Given any EZ-complex $K$ we get a contravariant functor $L=$ $L_{K}: \widetilde{\Delta} \rightarrow$ Set given by $L([n]):=K_{n}$ for all $n \in \mathbb{N}$ and, for any $\mu:[n] \rightarrow[n+m]$ write

$$
\mu=\delta_{k_{m-1}}^{n+m-1} \circ \cdots \circ \delta_{k_{1}}^{n+1} \circ \delta_{k_{0}}^{n}
$$

and define

$$
L(\mu)=\partial_{k_{0}}^{n-1} \circ \partial_{k_{1}}^{n} \circ \cdots \circ \partial_{k_{m-1}}^{n+m-2} .
$$

Also set $L\left(1_{[n]}\right)=1_{[n]}$. 
Proof. The proof follows by remarking that all morphisms in $\widetilde{\Delta}$ are of the form $\mu:[n] \rightarrow[n+m]$ for some $n, m \in \mathbb{N}$. For $\nu:[n+m] \rightarrow[n+m+l]$, the equation $L(\nu \circ$ $\mu)=L(\mu) \circ L(\nu)$ follows from Lemma 3.13.

Given Propositions 3.12 and 3.14 we can identify an EZ-complex with a contravariant functor from $\widetilde{\Delta}$ to Set and vice-versa. A morphism of EZ-complexes is then a natural transformation between the associated contravariant functors. Denote the category of EZ-complexes by EZ-Comp.

For the next lemma recall that $\iota_{k}^{n}: \Delta^{n-1} \rightarrow \Delta^{n}$ is the face inclusion associated to the unique $(n-1)$-face of $\Delta^{n}$ which does not contain the $k$ th vertex of $\Delta^{n}$. Note that $\iota_{k}^{n}\left(e_{i}\right)=e_{\delta_{k}^{n-1}(i)}$ on the vertices $\left\{e_{1}, \ldots, e_{n}\right\}$ of $\Delta^{n-1}$. So $\iota_{k}^{n}$ is "formally" the same as $\delta_{k}^{n-1}$.

Lemma 3.15. For any $n \in \mathbb{N}$ and any $1 \leqslant i<j \leqslant n+1$ the following identity holds:

$$
\iota_{j}^{n+1} \circ \iota_{i}^{n}=\iota_{i}^{n+1} \circ \iota_{j-1}^{n} .
$$

Proof. This is routine to check. Formally (as remarked above) this is same as Lemma 3.11 except for the indexing.

Definition 3.16. Given a $\Delta$-complex, $\mathcal{X}$, let

$$
\mathfrak{K}(\mathcal{X})_{n}=\left\{\sigma_{\alpha} \in \Sigma: \operatorname{Dom}\left(\sigma_{\alpha}\right)=\Delta^{n}\right\}=\Sigma_{n}
$$

for each $0<n \in \mathbb{N}$ and

$$
\partial_{i}^{n}\left(\sigma_{\alpha}\right)=\sigma_{\alpha} \circ \iota_{i}^{n}: \Delta^{n-1} \rightarrow X
$$

is the 'pre-compose by the inclusion of the $i$ th face' function for all $0<n \in \mathbb{N}$ and all $1 \leqslant i \leqslant n$.

We remark that one can think of $\mathfrak{K}(\mathcal{X})$ as a subset of $D_{\mathcal{X}}$.

Proposition 3.17. Let $\mathfrak{K}(\mathcal{X})$ be the collections of sets $\mathfrak{K}(\mathcal{X})_{n}$ together with the collection of maps $\partial_{i}^{n}$ as defined in Definition 3.16. Then $\mathfrak{K}(\mathcal{X})$ is an EZ-complex.

Proof. Notice that whenever $1 \leqslant i<j \leqslant n+1$, for any $n \in \mathbb{N}$,

$$
\begin{aligned}
\partial_{i}^{n-1} \partial_{j}^{n}\left(\sigma_{\alpha}\right) & =\partial_{i}^{n-1}\left(\sigma_{\alpha} \circ \iota_{j}^{n}\right) \\
& =\sigma_{\alpha} \circ \iota_{j}^{n} \circ \iota_{i}^{n-1} \\
& =\sigma_{\alpha} \circ \iota_{i}^{n} \circ \iota_{j-1}^{n-1} \\
& =\partial_{j-1}^{n-1} \partial_{i}^{n}\left(\sigma_{\alpha}\right)
\end{aligned}
$$

hence $\mathfrak{K}(\mathcal{X})$ is an EZ-complex as claimed.

Definition 3.18. Given a morphism $f: \mathcal{X} \rightarrow \mathcal{Y}$ of $\Delta$-complexes, define

$$
\mathfrak{K}(f): \mathfrak{K}(\mathcal{X}) \rightarrow \mathfrak{K}(\mathcal{Y})
$$

to be the pointwise post-composition function

$$
\mathfrak{K}(\mathcal{X})_{n} \ni \sigma_{\alpha} \longmapsto f \circ \sigma_{\alpha} .
$$

That this is well-defined is exactly the definition of a morphism of $\Delta$-complexes. 
Proposition 3.19. The assignment of Definition 3.18 is a (covariant) functor

\section{$\mathfrak{K}: \Delta$-Comp $\rightarrow$ EZ-Comp .}

Proof. Given a morphism $f: \mathcal{X} \rightarrow \mathcal{Y}$ in $\boldsymbol{\Delta}$-Comp by definition we have that $\mathfrak{K}(f)$ is a morphism in EZ-Comp from $\mathfrak{K}(\mathcal{X})$ to $\mathfrak{K}(\mathcal{Y})$, thus domains and codomains are respected as required.

Further, since $\mathfrak{K}\left(1_{X}\right)_{n}$ is the 'post-compose with the identity' function it is clear that $\mathfrak{K}\left(1_{\mathcal{X}}\right)$ is the identity morphism.

Finally, given morphisms $f: \mathcal{X} \rightarrow \mathcal{Y}$ and $g: \mathcal{Y} \rightarrow \mathcal{Z}$ in $\Delta$-Comp we compute

$$
\mathfrak{K}(g \circ f)\left(\sigma_{\alpha}\right)=(g \circ f) \circ \sigma_{\alpha}=g \circ\left(f \circ \sigma_{\alpha}\right)=\mathfrak{K}(g)\left(f \circ \sigma_{\alpha}\right)=\mathfrak{K}(g) \circ \mathfrak{K}(f)\left(\sigma_{\alpha}\right) .
$$

Definition 3.20 (MacLane $[\mathbf{1 6}]$ ). Let $\mathcal{C}$ and $\mathcal{D}$ be two categories and let $F: \mathcal{C} \rightarrow \mathcal{D}$ be a functor. Then $F$ induces a function

$$
F_{X, Y}: \operatorname{hom}_{\mathcal{C}}(X, Y) \rightarrow \operatorname{hom}_{\mathcal{D}}(F(X), F(Y))
$$

for every pair of objects $X$ and $Y$ in $\mathcal{C}$. The functor $F$ is said to be

- fully faithful if $F_{X, Y}$ is bijective for each $X$ and $Y$ in $\mathcal{C}$.

- essentially surjective if each object $d$ in $\mathcal{D}$ is isomorphic to an object of the form $F c$, for $c$ in $\mathcal{C}$.

Theorem 3.21. The functor $\mathfrak{K}$ is an equivalence of categories between $\boldsymbol{\Delta}$-Comp and EZ-Comp.

Proof. Recall (see MacLane [16, Theorem IV.4.1 (iii)]) that two categories $\mathbb{X}$ and $\mathbb{Y}$ are equivalent if and only if there is a functor $F: \mathbb{X} \rightarrow \mathbb{Y}$ such that $F$ is fully faithful and essentially surjective. Thus we need to show that $\mathfrak{K}$ is fully faithful and essentially surjective.

To show that $\mathfrak{K}$ is faithful let $f, g: X \rightarrow Y$ be distinct morphisms in $\boldsymbol{\Delta}$-Comp. We want to show that $\mathfrak{K}(f) \neq \mathfrak{K}(g)$ in EZ-Comp. Since $f \neq g$ there is a point $x \in X$ such that $f(x) \neq g(x)$ by the definition of a function. Since $X$ is a $\Delta$-complex we get that there is an $\alpha$ such that $x$ is an element of the image of $\sigma_{\alpha}: \Delta^{n_{\alpha}} \rightarrow X$, hence $f \circ \sigma_{\alpha} \neq g \circ \sigma_{\alpha}$. Then

$$
\mathfrak{K}(f)\left(\sigma_{\alpha}\right)=f \circ \sigma_{\alpha} \neq g \circ \sigma_{\alpha}=\mathfrak{K}(g)\left(\sigma_{\alpha}\right)
$$

so $\mathfrak{K}(f) \neq \mathfrak{K}(g)$.

To show that $\mathfrak{K}$ is full, let $\tilde{f}: \mathfrak{K}(\mathcal{X}) \rightarrow \mathfrak{K}(\mathcal{Y})$ be a morphism in EZ-Comp, where $\mathcal{X}=(X, \Sigma)$ and $\mathcal{Y}=\left(Y, \Sigma^{\prime}\right)$. Recall from Lemma 3.7 that $\mathcal{X}=\operatorname{colim} D_{\mathcal{X}}$ and $\mathcal{Y}=$ colim $D_{\mathcal{Y}}$. Thus, to define a morphism from $\mathcal{X}$ to $\mathcal{Y}$, it suffices by Remark 3.8 to specify a functor $F: D_{\mathcal{X}} \rightarrow D_{\mathcal{Y}}$. Define

$$
F\left(\Delta^{n_{\alpha}}, \sigma_{\alpha}\right)=\left(\Delta^{n_{\alpha}}, \tilde{f}\left(\sigma_{\alpha}\right)\right)
$$

and for any face inclusion $\iota: \Delta^{n_{\alpha}} \rightarrow \Delta^{n_{\beta}}$ define

$$
F(\iota)=\tilde{f}\left(\sigma_{\beta}\right) \circ \iota: \Delta^{n_{\alpha}} \rightarrow Y .
$$


For $\iota_{j}^{n_{\beta}}: \Delta^{n_{\beta}-1} \rightarrow \Delta^{n_{\beta}}$, we have

$$
\begin{aligned}
F\left(\iota_{j}^{n_{\beta}}\right)=\tilde{f}\left(\sigma_{\beta}\right) \circ \iota_{j} & =\partial_{j}\left(\tilde{f}\left(\sigma_{\beta}\right)\right) \\
& =\tilde{f}\left(\partial_{j}\left(\sigma_{\beta}\right)\right) \quad \text { Definition } 3.16 \\
& =\tilde{f}\left(\sigma_{\beta} \circ \iota_{j}\right) \\
& =\tilde{f}\left(\sigma_{\alpha}\right) .
\end{aligned}
$$

Since any face inclusion $\iota: \Delta^{m} \hookrightarrow \Delta^{n}$ is a composition $\iota=\iota_{k_{n-m}}^{n-1} \circ \cdots \circ \iota_{k_{1}}^{m}$, the above equality and the associative law show $\tilde{f}\left(\sigma_{\beta}\right) \circ \iota=\tilde{f}\left(\sigma_{\alpha}\right)$ (where $m=n_{\alpha}$ and $n=n_{\beta}$ ), hence the collection $\tilde{f}\left(\sigma_{\beta}\right)$ forms a cocone from the base $D$ into $Y$. The universal property of the colimit then gives a morphism $f=\operatorname{colim} F: X \rightarrow Y$ such that $f \circ$ $\sigma_{\alpha}=\tilde{f}\left(\sigma_{\alpha}\right)$ for all $\sigma_{\alpha} \in \Sigma$. But

$$
\mathfrak{K}(f)_{n}\left(\sigma_{\alpha}\right)=f \circ \sigma_{\alpha}=\tilde{f}\left(\sigma_{\alpha}\right)
$$

by definition, hence $\mathfrak{K}(f)=\tilde{f}$. Since $\tilde{f}$ was arbitrary we get that $\mathfrak{K}$ is full.

To show that $\mathfrak{K}$ is essentially surjective pick any EZ-complex $K: \widetilde{\Delta} \rightarrow$ Set. We will construct a $\Delta$-complex $\mathcal{X}$ such that $\mathfrak{K}(\mathcal{X}) \cong K$. This is motivated by the similar construction in $[\mathbf{1 1}$, p. 104]. First we define a diagram in $\mathbf{\Delta}$-Comp in the following way. Define the objects of $D$ to be, for each $n \in \mathbb{N}$ and each $\alpha \in K_{n}$, a copy of $\Delta^{n_{\alpha}}$ indexed by $\alpha$. For each $n \in \mathbb{N}$, each integer $m \geqslant 1$, each $\alpha \in K_{n+m}$, and every morphism $\gamma:[n] \rightarrow[n+m]$ in $\widetilde{\Delta}$ write

$$
\gamma=\delta_{k_{m-1}}^{n+m-1} \circ \cdots \circ \delta_{k_{1}}^{n+1} \circ \delta_{k_{0}}^{n}
$$

and set

$$
D(K(\gamma)(\alpha), \alpha):=\left\{\iota_{k_{m-1}}^{n+m} \circ \cdots \circ \iota_{k_{0}}^{n+1}\right\}
$$

where $K(\gamma): K_{n+m} \rightarrow K_{n}$. These sets are composition-closed with respect to the index by functoriality. Construct

$$
X=\left(\bigsqcup \Delta^{n_{\alpha}}\right) / \sim
$$

where $\sim$ is the same set of identifications mentioned in Hatcher [11, p. 104]. It is routine to check that

$$
\left(X,\left\{\phi_{\alpha}: \Delta^{n_{\alpha}} \rightarrow X \text { and } \alpha \in \bigcup_{n \in \mathbb{N}} K_{n}\right\}\right)
$$

is a $\Delta$-complex, that is, it satisfies the conditions $\mathrm{A} 1, \ldots, \mathrm{A} 4$ of Definition 3.2.

Further $\mathfrak{K}(\mathcal{X}) \cong K$ since each $\mathfrak{K}(\mathcal{X})_{n}$ consists of exactly one face inclusion for each element of $K_{n}$, and since, for each $n \in \mathbb{N}$ and each $k \leqslant n$ we have, in $\mathfrak{K}(\mathcal{X})$, that

$$
\partial_{k}^{n}\left(\phi_{\alpha}\right)=\phi_{\alpha} \iota_{k}^{n}=\phi_{\partial_{k}^{n}(\alpha)}
$$

by the construction of the diagram $D$. Thus the morphism $K \rightarrow \mathfrak{K}(\mathcal{X})$ defined by sending $\alpha$ to $\phi_{\alpha}$ is both well-defined and a pointwise isomorphism. Natural transformations which are pointwise isomorphisms are natural isomorphisms, hence $K \cong \mathfrak{K}(\mathcal{X})$ in EZ-Comp. We conclude that $\mathfrak{K}$ is essentially surjective.

The next corollary deals with the chain complex functors from either of these two categories to Comp, the category of chain complexes of abelian groups. 
Corollary 3.22. The functor $\mathfrak{K}$ commutes with the chain complex functors on $\Delta$-Comp and EZ-Comp, indeed one has the commutative diagram

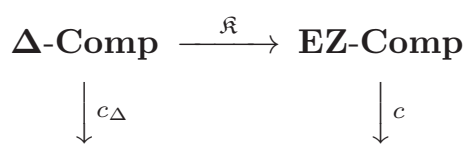

\section{Comp $=$ Comp.}

Proof. For any $\Delta$-complex $\mathcal{X}=(X, \Sigma)$, we find that both $C_{n}(\mathcal{X})$ and $C_{n}(\mathfrak{K}(\mathcal{X}))$ are equal to the free abelian group on $\Sigma_{n}=\mathfrak{K}(\mathcal{X})_{n}$. Since $\partial_{i}^{n}\left(\sigma_{\alpha}\right)=\sigma_{\alpha} \circ \iota_{i}^{n}$, as in Definition 3.16, the boundary maps are also identical.

From Corollary 3.22 and [21, Theorem 1.7, Proposition 2.1] we have the following.

Corollary 3.23. The categories $\Delta$-Comp, EZ-Comp and Simp are equivalent.

Remark 3.24. As proved in $[\mathbf{1 1}]$, the natural inclusion of the chains of a $\Delta$-complex $X$ into the singular chains $S(X)$ induces an isomorphism in homology. Therefore the homology of $\Delta$-complexes is a homology theory on $\boldsymbol{\Delta}$-Comp satisfying the EilenbergSteenrod axioms.

\section{Construction of $\Delta$-complexes}

We commence this section by proving a basic result, Theorem 4.1, which says that a $G$-action on a $\Delta$-complex $X$ by $\Delta$-morphisms induces a $\Delta$-complex structure on $|X| / G$. This is followed by a few definitions and remarks pertaining to the case where $X$ is a simplicial complex. Then, after giving some further results, we compare our Definition 4.3 with the corresponding definitions of Illman [14] using an example. Throughout the section, it is assumed that $G$ and $X$ are finite.

Theorem 4.1. Let $\mathcal{X}=(X, \Sigma)$ be a $\Delta$-complex and $G$ act on $\mathcal{X}$ by $\Delta$-morphisms. Then the orbit space $X / G$ has a $\Delta$-complex structure $(X / G, \bar{\Sigma})$ and the canonical identification map $\pi: X \rightarrow X / G$ induces a morphism $(X, \Sigma) \rightarrow(X / G, \bar{\Sigma})$ of $\Delta$ complexes.

Proof. We adhere to the notations of Section 3. Since $G$ acts on $\mathcal{X}$ by $\Delta$-morphisms, if $\sigma: \Delta^{n} \rightarrow X$ is an $n$-simplex then the composition $g \sigma$ given by $\Delta^{n_{\alpha}} \stackrel{\sigma}{\rightarrow} X \stackrel{g}{\rightarrow} X$ is an $n$-simplex in $\mathcal{X}$. That is, if $\sigma \in \Sigma_{n}$ then $g \sigma \in \Sigma_{n}$. Conversely, if $g \sigma=\tau \in \Sigma_{n}$ then $\sigma=g^{-1} \tau \in \Sigma_{n}$ for all $g \in G$. Thus $X^{n}$ is a $G$-invariant subset of $X$, where $X^{n}$ is the union of the images of all $\sigma \in \Sigma_{m}, m \leqslant n$, i.e., the $n$-skeleton of $X$. Let $\sigma \in \Sigma_{n}$. Then

$$
\pi^{-1}\left(\pi\left(\sigma\left(\Delta^{n}\right)\right)\right)=\bigcup\left\{g \sigma\left(\Delta^{n}\right): g \in G\right\} .
$$

Suppose $g \sigma\left(\Delta^{n}\right) \cap \sigma\left(\Delta^{n}\right) \neq \emptyset$ and $x$ belongs to this intersection. So $x$ is in the image of $g \stackrel{\circ}{\sigma}$ and $\stackrel{\circ}{\sigma}$. Then by condition A2 of Definition 3.2, $g \sigma\left(\Delta^{n}\right)=\sigma\left(\Delta^{n}\right)$. Since the $G$-action on $\mathcal{X}$ preserves the ordering of each simplex, $g \sigma(x)=\sigma(x)$ for all $x \in \Delta^{n}$. 
Summarizing the above, either $g \sigma\left(\Delta^{n}\right) \cap \sigma\left(\Delta^{n}\right)=\emptyset$ or $\left.g\right|_{\sigma\left(\Delta^{n}\right)}=i d_{\sigma\left(\Delta^{n}\right)}$. From this observation and (2) it follows that

$$
\left.\pi\right|_{\sigma\left(\Delta^{n}\right)}: \sigma\left(\Delta^{n}\right) \rightarrow \pi\left(\sigma\left(\Delta^{n}\right)\right)
$$

is a homeomorphism. On the other hand, any $G$-action on $\mathcal{X}$ by $\Delta$-morphisms induces an equivalence relation on $\Sigma$ where as usual $\sigma \sim \tau$ if and only if $\tau=g \sigma$ for some $g \in G$. Let $A_{n}=\left\{\sigma_{n 1}, \ldots, \sigma_{n k_{n}}\right\}$ be a set of representatives for the equivalence classes of $\sigma \in \Sigma_{n}$.

So $\sigma_{n i}$ 's are distinct and

$$
X / G=\bigcup_{n=0}^{\operatorname{dim}(X)} \bigcup_{\sigma \in A_{n}} \pi\left(\sigma\left(\Delta^{n}\right)\right) .
$$

It is now straightforward that the collection of maps

$$
\beta_{n i}=\pi \circ \sigma_{n i}: \Delta^{n} \rightarrow X / G,
$$

where $0 \leqslant n \leqslant \operatorname{dim}(K)$ and $1 \leqslant i \leqslant k_{n}$, satisfies the conditions of Definition 3.2 of a $\Delta$-complex. Therefore there is a $\Delta$-complex structure on $X / G$ with vertices $V(X) / G$ and clearly the identification map is a morphism of $\Delta$-complexes.

Definition 4.2. 1. A (geometric) simplicial complex $K$ is a finite collection of simplexes in some $\mathbb{R}^{N}$ satisfying: (i) if $\sigma_{1}$ is a face of $\sigma_{2}$ and $\sigma_{2} \in K$ then $\sigma_{1} \in K$, (ii) if $\sigma_{1}, \sigma_{2} \in K$ then $\sigma_{1} \cap \sigma_{2}$ is a face of both $\sigma_{1}$ and $\sigma_{2}$. The zero dimensional cells of $K$ are called the vertices $V(K)$ of $K$ and the empty set $\emptyset$ is a $(-1)$-simplex by convention. The dimension of $K$ is the maximum of the dimensions of the simplexes in $K$.

2. For any simplicial complex $K$ and any subset $L \subseteq K$, one defines its polyhedron as

$$
|L|=\bigcup_{\sigma \in L} \sigma \subset \mathbb{R}^{N}
$$

Note that $|L|$ is a compact subset of $\mathbb{R}^{N}$. Since we wish to work in the category $\Delta$-Comp, we shall usually assume that the vertices of any simplex of $K$ are simply ordered so that if $\sigma_{1}$ is a face of $\sigma_{2}$ then the ordering of the vertices of $\sigma_{1}$ agrees with the ordering coming from $\sigma_{2}$, and in this case call $K$ an ordered simplicial complex. For instance, Example 3.6 gives a method to obtain such an ordering from a partial ordering on $V(K)$, but note that orderings that are not of this type also exist (cf. Example 4.12). Next we introduce the idea of a $G$-action on a (finite) simplicial complex.

Definition 4.3. Let $G$ be a finite group and $K$ be a finite simplicial complex. A $G$-action on $K$ is called simplicial if

$$
\sigma=\left\langle v_{0}, \ldots, v_{r}\right\rangle \in K \quad \text { then } \quad g \sigma=g(\sigma)=\left\langle g v_{0}, \ldots, g v_{r}\right\rangle \in K
$$

In addition, if $K$ is an ordered simplicial complex and $G$ preserves the ordering of each simplex of $K$, we call $K$ an ordered simplicial $G$-complex. 
Example 4.4. Let $v_{0}=-1, v_{1}=1$ and $\left\langle v_{0}, v_{1}\right\rangle=[-1,1]$. Then $K=\left\{\left\langle v_{0}\right\rangle,\left\langle v_{1}\right\rangle\right.$, $\left.\left\langle v_{0}, v_{1}\right\rangle\right\}$ is a simplicial complex. The order two flip $(x \mapsto-x)$ on $\left\langle v_{0}, v_{1}\right\rangle$ is a simplicial action of $\mathbb{Z}_{2}$ on $K$ but not an ordered simplicial action.

Remark 4.5. In $[\mathbf{1 4}, \S 1]$ the definitions of both a simplicial $G$-complex, and an equivariant simplicial $G$-complex, are given. At the end of this section (Example 4.12) we compare these definitions with Definition 4.3. Note that Definition 4.3 induces a $G$-action on the polyhedron $|K|$ via

$$
\sum_{i=0}^{n} t_{i} v_{i} \longmapsto \sum_{i=0}^{n} t_{i} g\left(v_{i}\right)
$$

and we denote both the actions by the same notation. This action is clearly a simplicial homeomorphism for each $g \in G$.

Our primary concern in this section is quotients of ordered simplicial complexes, and, in particular, we get the following as a special case of Theorem 4.1.

Proposition 4.6. Let $K$ be an ordered simplicial $G$-complex. Then the orbit space $|K| / G$ is a $\Delta$-complex with vertices $V(K) / G$. Furthermore, the canonical identification map $\pi:|K| \rightarrow|K| / G$ is a morphism of $\Delta$-complexes.

Proposition 4.7. Let $K$ be a simplicial complex and $\tau$ a free simplicial involution on $K$, i.e., a free $\mathbb{Z}_{2}$-action on $K$. Then there exists an ordering of the vertices of $K$ such that $K$ is an ordered simplicial $\mathbb{Z}_{2}$-complex.

Proof. Let $V$ be the set of vertices of $K$. One can write $V=A \sqcup \tau A$, where $A$ is a subset of $V$ containing exactly half of the vertices of $V$. Let $v_{1}<v_{2}<\cdots<v_{k}$ be an order on $A$. Define an order on $V$ by

$$
v_{1}<\tau v_{1}<v_{2}<\tau v_{2}<\cdots<v_{k}<\tau v_{k} .
$$

This induces a $\Delta$-complex structure on $K$, which we also denote by $K$. Since $K$ is a simplicial complex and $\tau$ is a free involution, $\left\langle v_{i}, \tau v_{i}\right\rangle$ is not an edge in $K$. Let $\sigma=\left\langle u_{1}, \ldots, u_{\ell}\right\rangle \in K$. Then $u_{i}$ is either $v_{j_{i}}$ or $\tau v_{j_{i}}$ for $i=1, \ldots, \ell$ and $j_{i}<j_{i+1}$ for $i=1, \ldots, \ell-1$. So $\tau u_{i}$ is respectively either $\tau v_{j_{i}}$ or $v_{j_{i}}$. Then

$$
\tau \sigma=\left\langle\tau u_{1}, \ldots, \tau u_{\ell}\right\rangle \text { and } \tau u_{1}<\cdots<\tau u_{\ell} .
$$

Therefore $\tau$ preserves the order on each simplex of $K$. This proves the proposition.

Propositions 4.6 and 4.7 imply the following.

Corollary 4.8. For any free involution $\tau$ on a finite simplicial complex $K$, the space $|K| / \mathbb{Z}_{2}$ has a $\Delta$-complex structure.

Next we define a simplicial $G$-complex and an equivariant simplicial $G$-complex following Illman [14], and we compare these definitions with our Definition 4.3 by means of Proposition 4.11 and Example 4.12.

Definition 4.9 (Illman [14]). Let $K$ be a simplicial complex and $G$ act on $|K|$. Then $K$ is called a simplicial $G$-complex, if the map $g: K \rightarrow K$ is a simplicial homeomorphism for all $g \in G$. Moreover, $K$ is called an equivariant simplicial $G$-complex if the following also holds: 
1. if $\left\langle v_{0}, \ldots, v_{k}\right\rangle \in K$ and $\left\langle g_{0} v_{0}, \ldots, g_{k} v_{k}\right\rangle \in K$ for some $g_{0}, \ldots, g_{k} \in G$, then there exists $g \in G$ such that $g_{i} v_{i}=g v_{i}$ for $i=0, \ldots, k$. Here $v_{0}, \ldots, v_{k}$ need not be distinct.

2. any simplex $\left\langle v_{0}, \ldots, v_{k}\right\rangle \in K$ can be ordered such that the inclusions $G_{v_{k}} \subseteq$ $\cdots \subseteq G_{v_{0}}$ hold for the isotropy groups.

In the next remark observations 2 and 3 are due to Illman, and given in [14] without proof.

Remark 4.10. 1. An equivariant simplicial $G$-complex is trivially a simplicial $G$ complex. Also, by the final sentence of Remark 4.5, an ordered simplicial $G$ complex is a simplicial $G$-complex.

2. The identification space of an equivariant simplicial $G$-complex by the action of $G$ is a simplicial complex.

3. For any simplex $\sigma$, if $x \in \sigma$ and $g x \in \sigma$ then $g x=x$, and if $x \in \stackrel{\circ}{\sigma}$ and $g x=x$ then $g y=y$ for all $y \in \sigma$.

Proposition 4.11. Let $K$ be an equivariant simplicial $G$-complex. Then there exists an ordering for the simplexes of $K$ with respect to which $K$ is an ordered simplicial G-complex.

Proof. By Remark 4.10 (2) $K / G$ is a simplicial complex. Writing the vertices of $K$ as $v$ and of $K / G$ as $[v]$, we can then order the vertices $[v] \in K / G$ using a suitable partial order $\preceq$ as in Example 3.6. Lifting this to a partial order $\leqslant$ for the vertices of $K$, i.e. $v_{1} \leqslant v_{2}$ if and only if $[v]_{1} \preceq\left[v_{2}\right]$, then $\leqslant$ has the property that the vertices of any simplex of $K$ are simply ordered, and it is preserved under the $G$-action.

We next give an example of an ordered simplicial $G$-complex which is not an equivariant simplicial $G$-complex. So, the category of ordered simplicial $G$-complexes properly contains the category of equivariant simplicial $G$-complexes.

Example 4.12. In this example the arrows on the edges imply the ordering of simplexes. For example, the 2-simplex $v_{0} v_{1} v_{2}$ in Figure 1 is ordered as $\left\langle v_{1}, v_{2}, v_{0}\right\rangle$.

Consider the 2-dimensional simplicial complex $K$ corresponding to Figure 1 . The

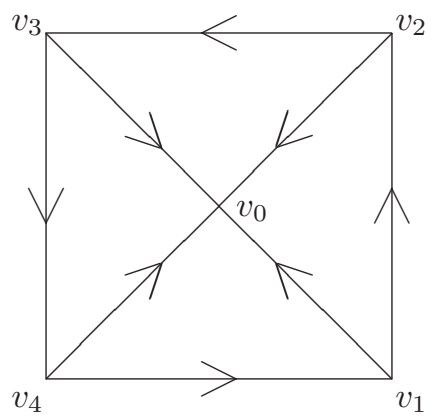

Figure 1: An ordered simplicial $G$-complex. 
cyclic group $\mathbb{Z}_{4} \cong G=\left\langle\alpha \mid \alpha^{4}=1\right\rangle$ acts on $K$ as determined by

$$
\alpha v_{0}=v_{0}, \alpha v_{4}=v_{1} \text {, and } \alpha v_{i}=v_{i+1} \text { for } i=1,2,3 .
$$

Then $v_{0}$ is fixed by $G$. Notice that the $G$-action is order preserving. So $K$ is an ordered simplicial $G$-complex. Thus by Proposition $4.6,|K| / G$ is a $\Delta$-complex with vertices $V(K) / G$.

Observe that the $G$-action on $K$ does not satisfy Remark 4.10 (2) or Remark 4.10 (3), so this is not an equivariant simplicial $G$-complex.

In the above we discussed group actions which preserve the ordering of each simplex. More generally we may ask the following.

Question: Let $K$ be simplicial $G$-complex. Do there exist any other (possibly more general) sufficient conditions which ensure that $|K| / G$ has the structure of a $\Delta$-complex?

\section{Application to real projective space}

A fairly simple way to triangulate $\mathbb{R} P^{n}$ is to start with the standard triangulation of $S^{n}$ as the boundary of an $n$-simplex. The antipodal map $\tau$ is then not a simpicial map, however, if one passes to the first barycentric subdivision the antipodal map is now simplicial with $\sigma \cap \tau \sigma=\emptyset$ for all simplexes, hence a triangulation of $\mathbb{R} P^{n}=$ $S^{n} / \mathbb{Z}_{2}$ is induced with $2^{n+1}-1$ vertices, see Example 3.20 in [5]. Also, Basak and the second author constructed some triangulation of $\mathbb{R P}^{n}$ with $2^{n}+n+1$ vertices, see Theorem 3.24 in [1]. Using these triangulations to compute the cohomology ring of $\mathbb{R} P^{n}$ still seems very complicated (the authors succeeded for $n \leqslant 3$ ).

However, $\mathbb{R} P^{n}$ admits a much simpler structure as a $\Delta$-complex with only $n+1$ vertices, already mentioned as an exercise in [11]. After setting up some notation, we shall show how this $\Delta$-complex can be used to give an elementary and explicit computation of the cohomology ring. A similar computation is discussed in $[\mathbf{1 2}]$ using an alternative technique, block-dissections.

Following Coxeter $[\mathbf{3}]$, the regular polytope $\beta_{n} \subset \mathbb{R}^{n+1}$ with the $2(n+1)$ vertices $\pm e_{i}, 1 \leqslant i \leqslant n+1$, is called the $n$-dimensional cross polytope (or orthoplex), and is homeomorphic to $S^{n}$. For $n=1,2$ it is respectively the familiar square, octahedron. It will be useful to label the vertices as $v_{i}=e_{i}, w_{i}=-e_{i}$. Of course $\beta_{n}$ is a simplicial complex, with vertex set

$$
V=\left\{v_{i}, w_{i}: 1 \leqslant i \leqslant n+1\right\}=\left\{\lambda v_{1}, \ldots, \lambda v_{n+1}: \lambda= \pm 1\right\} .
$$

Any subset of $V$ that contains no pair $v_{i}, w_{i}$ will form the vertices of a simplex of the simplicial complex $\beta_{n}$. Equivalently, an $r$-simplex of $\beta_{n}$ can be written as $\sigma=\left\langle\lambda_{0} v_{i_{0}}, \ldots, \lambda_{r} v_{i_{r}}\right\rangle$, where $1 \leqslant i_{0}<i_{1}<\ldots<i_{r} \leqslant n+1, \lambda_{i}= \pm 1$ and $\left\langle x_{0}, \ldots x_{r}\right\rangle$ denotes the convex hull of points $x_{0}, \ldots, x_{r} \in \mathbb{R}^{n+1}$. From this description we see that the number of $r$-simplexes equals $2^{r+1} \cdot\left(\begin{array}{c}n+1 \\ r+1\end{array}\right)$.

Now let $\tau$ be the simplicial map of $\beta_{n}$ to itself induced by defining $\tau\left(\lambda v_{i}\right)=-\lambda v_{i}$ on the vertices of $\beta_{n}$. Clearly $\tau$ is a simplicial involution and gives rise to a free simplicial action of the group $C_{2}=\{e, \tau\}$ on $\beta_{n}$. Also note $\left|\beta_{n}\right| \cong S^{n}$ with $\tau(x)=-x$ 
for any $x \in\left|\beta_{n}\right|$, hence

$$
\left|\beta_{n}\right| / C_{2} \cong \mathbb{R} P^{n}
$$

Each $r$-simplex $\sigma$ of $\mathbb{R} P^{n}$ can be written as

$$
\sigma=\left\langle x_{i_{0}}, x_{i_{1}}, \ldots, x_{i_{r}}\right\rangle=\left\langle-x_{i_{0}},-x_{i_{1}}, \ldots,-x_{i_{r}}\right\rangle,
$$

where $1 \leqslant i_{0}<i_{1}<\ldots<i_{r} \leqslant n+1$ and $x_{i_{j}} \in\left\{v_{i_{j}}, w_{i_{j}}\right\}, 0 \leqslant j \leqslant r$ (as before $v_{i_{j}}=$ $\left.e_{i_{j}}, w_{i_{j}}=-e_{i_{j}}\right)$. This notation is compatible with the simple order $v_{1}<w_{1}<v_{2}<$ $\cdots<v_{n+1}<w_{n+1}$ of the vertices of $\beta_{n}$ so gives both $\beta_{n}$ and $\mathbb{R} P^{n}$ a $\Delta$-complex structure (which would also arise by applying Proposition 4.6 or Corollary 4.8).

We now turn our attention to describing the chain complex $\mathcal{C}$ arising from this $\Delta$-complex, and then using it to give an elementary computation of the cohomology ring $H^{*}\left(\mathbb{R} P^{n} ; \mathbb{Z}_{2}\right)$. Of course this is well known to be the truncated polynomial algebra $\mathbb{Z}_{2}[u] /\left(u^{n+1}=0\right)$ and can be found in many texts, e.g. $[\mathbf{1 1}]$, but using more sophisticated machinery. Our goal here is to utilize the above $\Delta$-complex structure for $\mathbb{R} P^{n}$ to compute its homology and cohomology (ring) as well as to exhibit explicit generators for these, in terms of the simplexes of $\mathbb{R} P^{n}$.

In particular, the following notation for the $2^{n} n$-simplexes of $\mathbb{R} P^{n}$ will be convenient. From the above discussion each such simplex can be uniquely written $\sigma=$ $\left\langle x_{1}, \ldots, x_{n+1}\right\rangle$ where $x_{i} \in\left\{v_{i}, w_{i}\right\}$ and (for uniqueness) $x_{n+1}=v_{n+1}$. Equivalently,

$$
\sigma=\left\langle\lambda_{1} v_{1}, \ldots, \lambda_{n} v_{n}, v_{n+1}\right\rangle, \quad \lambda_{i}= \pm 1 .
$$

Then these $2^{n}$ simplexes can be written $\sigma_{A}$, one for each $A \subseteq \underline{n}=\{1,2, \ldots, n\}$, where $A=\left\{i: \lambda_{i}=-1\right\}$. For example, $\sigma_{\emptyset}=\left\langle v_{1}, v_{2}, \ldots, v_{n+1}\right\rangle$. The coefficients of each chain complex will be $\mathbb{Z}$ unless otherwise indicated, and $|A|$ will denote the cardinality of $A$. We remark that there is a slight abuse of notation here since we are using the same notation for the simplex $\sigma$ in $S^{n}$ and its image in $\mathbb{R} P^{n}$, but there should be no confusion since we only deal with $\mathbb{R} P^{n}$.

We first consider the chain complex $C_{*}\left(\mathbb{R} P^{n}, \mathbb{R} P^{n-1}\right)$ and its differential

$$
\tilde{d}: C_{j}\left(\mathbb{R} P^{n}, \mathbb{R} P^{n-1}\right) \rightarrow C_{j-1}\left(\mathbb{R} P^{n}, \mathbb{R} P^{n-1}\right),
$$

in particular, for $j=n$.

Lemma 5.1. (a) $H_{n}\left(\mathbb{R} P^{n}, \mathbb{R} P^{n-1}\right) \approx \mathbb{Z}$, with generator $s_{n}:=\sum_{A \subseteq \underline{n}}(-1)^{|A|} \sigma_{A}$.

(b) For $0 \leqslant i \leqslant n-1, H_{i}\left(\mathbb{R} P^{n}, \mathbb{R} P^{n-1}\right)=0$.

Proof. (a) Note that $C_{n}\left(\mathbb{R} P^{n}, \mathbb{R} P^{n-1}\right)=C_{n}\left(\mathbb{R} P^{n}\right)$ since $C_{n}\left(\mathbb{R} P^{n-1}\right)=0$, and also that $C_{n+1}\left(\mathbb{R} P^{n}, \mathbb{R} P^{n-1}\right)=0$. It follows that

$$
H_{n}\left(\mathbb{R} P^{n}, \mathbb{R} P^{n-1}\right)=\operatorname{Ker}\left(\tilde{d}_{n}: C_{n}\left(\mathbb{R} P^{n}\right) \rightarrow C_{n-1}\left(\mathbb{R} P^{n}, \mathbb{R} P^{n-1}\right)\right) .
$$

Since each $(n-1)$-simplex of $\mathbb{R} P^{n} \backslash \mathbb{R} P^{n-1}$ occurs in the boundary of precisely two $\sigma_{A}$ 's, any $n$-cycle $x \in \operatorname{Ker}(\tilde{d})$ must be an integral multiple of $\sum_{A \subseteq \underline{n}} \lambda_{A} \cdot \sigma_{A}$ for some $\lambda_{A}= \pm 1$. Without loss of generality fix $\lambda_{\emptyset}=+1$, then all the other signs $\lambda_{A}$ are uniquely determined by the cycle condition $\tilde{d}(x)=0$. It then only remains to check that $\tilde{d}\left(s_{n}\right)=0$ since this will imply that $x=m \cdot s_{n}$ for some $m \in \mathbb{Z}$ and hence gives the conclusion of the lemma. To complete the proof then, consider any $n-1$ simplex 
$\omega$ of $\mathbb{R} P^{n} \backslash \mathbb{R} P^{n-1}$. It must have the form $\sigma_{A}$ with a single vertex $v_{j}$ missing, where $1 \leqslant j \leqslant n$. Then it occurs in the boundary of precisely two $\sigma_{A}$, one for which $j \notin A$ and one for which $j \in A$. The cardinalities of these two subsets $A$ differ by 1 , so the form of $s_{n}$ guarantees that $\omega$ occurs with opposite signs in $\tilde{d}\left(s_{n}\right)$ and thus $\tilde{d}\left(s_{n}\right)=0$.

(b) A short non-simplicial proof of (b) follows from Proposition 2.22 of Hatcher [11], namely $H_{i}\left(\mathbb{R} P^{n}, \mathbb{R} P^{n-1}\right) \approx \widetilde{H}_{i}\left(\mathbb{R} P^{n} / \mathbb{R} P^{n-1}\right) \approx \widetilde{H}_{i}\left(S^{n}\right)=0, i<n$. We also give a simplicial proof of this fact by means of an explicit contracting homotopy in the chain complex itself, but defer this to the Appendix so that we can continue with the homology calculations.

Using this lemma, the next step is to compute the connecting homomorphism

$$
\partial: H_{n}\left(\mathbb{R} P^{n}, \mathbb{R} P^{n-1}\right) \rightarrow H_{n-1}\left(\mathbb{R} P^{n-1}\right) .
$$

Since, as established in Section $3, \Delta$-complexes give rise to a homology theory, knowing this connecting homomorphism together with Lemma 5.1 will suffice to determine (by induction) the exact homology sequence of the pair $\left(\mathbb{R} P^{n}, \mathbb{R} P^{n-1}\right)$, hence, in particular, the (integral) homology of $\mathbb{R} P^{n}$ with explicit generators. The differential in the chain complex $C_{*}\left(\mathbb{R} P^{n}\right)$ will be denoted

$$
d: C_{j}\left(\mathbb{R} P^{n}\right) \rightarrow C_{j-1}\left(\mathbb{R} P^{n}\right)
$$

(in contrast with $\tilde{d}$ defined earlier). We may regard $s_{n}$ as an $n$-chain in $C_{n}\left(\mathbb{R} P^{n}, \mathbb{R} P^{n-1}\right)$ or in $C_{n}\left(\mathbb{R} P^{n}\right)$ since, as noted in the proof of Lemma 5.1 , these are equal.

Theorem 5.2. (a) $\partial\left(s_{n}\right)=\left(1+(-1)^{n}\right) s_{n-1}$,

(b)

$$
H_{i}\left(\mathbb{R} P^{n}\right)= \begin{cases}\mathbb{Z} & : \quad i=0 \text { and } i=n \text { odd } \\ \mathbb{Z}_{2} & : \quad 0<i<n \text { and } n \text { odd }, \\ 0 & : \text { otherwise. }\end{cases}
$$

Proof. (a) Since, as noted above, $j_{*}: C_{n}\left(\mathbb{R} P^{n}\right) \rightarrow C_{n}\left(\mathbb{R} P^{n}, \mathbb{R} P^{n-1}\right)$ is the identity map, we have

$$
\partial\left(s_{n}\right)=\left(i_{*}\right)^{-1} d\left(j_{*}\right)^{-1}\left(s_{n}\right)=\left(i_{*}\right)^{-1} d\left(s_{n}\right) .
$$

As shown in the proof of Lemma 5.1, any simplex containing the vertex $v_{n+1}$ vanishes in $d\left(s_{n}\right)$, so we need only consider the coefficients of simplexes of the form $\left\langle\lambda_{1} v_{1}, \ldots, \lambda_{n} v_{n}\right\rangle$ in $d\left(s_{n}\right)$. Write such a simplex (non-uniquely) as $\tau_{A}, A \subseteq \underline{n}$, noting that $\tau_{A}=\tau_{B}$ if and only if $B=\underline{n} \backslash A$. By adjoining the vertex $v_{n+1}$ to $\tau_{A}, \tau_{B}$ we obtain two distinct $n$-simplexes $\sigma_{A}, \sigma_{B}$ of $\mathbb{R} P^{n}$. Now $d\left(\sigma_{A}\right)$ will contain $\tau_{A}$ with coefficient $(-1)^{n+1} \cdot(-1)^{|A|}$ whereas $d\left(\sigma_{B}\right)$ will contain $\tau_{B}=\tau_{A}$ with coefficient

$$
(-1)^{n} \cdot(-1)^{|B|}=(-1)^{n} \cdot(-1)^{n-|A|} \text {. }
$$

For $n$ odd these two coefficients add to 0 , while for $n$ even they add to $2 \cdot(-1)^{|A|}$. Since in the $n$ even case this sum is precisely $i_{*}\left(2 s_{n-1}\right)$, and 0 in the odd case, (a) is proved.

(b) This is a straightforward induction, starting the induction with the known case $\mathbb{R} P^{1} \cong S^{1}$, and using the exact homology sequence of the pair $\left(\mathbb{R} P^{n}, \mathbb{R} P^{n-1}\right)$ together with (a) as well as Lemma 5.1 to complete the inductive step. Note that 
there are two cases in the inductive step, $(n-1)$ odd to $n$ even or vice-versa. We omit the easily verified details.

Applying the universal coefficient theorem, or repeating the above proof with $\mathbb{Z}_{2}$ coefficients, now gives the following corollary at once.

Corollary 5.3. For all $j, 0 \leqslant j \leqslant n$, one has $H_{j}\left(\mathbb{R} P^{n} ; \mathbb{Z}_{2}\right) \approx \mathbb{Z}_{2}$ and $H^{j}\left(\mathbb{R} P^{n} ; \mathbb{Z}_{2}\right) \approx$ $\mathbb{Z}_{2}$.

Using this corollary we can now turn to the final goal of this section, computing $H^{*}\left(\mathbb{R} P^{n} ; \mathbb{Z}_{2}\right)$ as a graded ring with explicit generator. We shall use the notation e.g. $\left\langle v_{1}, v_{2}, v_{3}\right\rangle^{*}$ to denote the dual cochain of the simplex $\left\langle v_{1}, v_{2}, v_{3}\right\rangle$ with coefficients $\mathbb{Z}$ or $\mathbb{Z}_{2}$, i.e. $\left\langle v_{1}, v_{2}, v_{3}\right\rangle^{*}(\sigma)=1$ if $\sigma=\left\langle v_{1}, v_{2}, v_{3}\right\rangle$ and is 0 otherwise, and similarly for any other $r$-simplex. Since cup products are to be considered, a suitable partial order of the vertices of the $\Delta$-complex must be used, we choose the (simple) order $v_{1}<$ $w_{1}<v_{2}<\ldots<v_{n+1}<w_{n+1}$. As with any connected $n$-manifold, any $n$ dimensional "co-simplex" $\left\langle\lambda_{1} v_{1}, \ldots, \lambda_{n+1} v_{n+1}\right\rangle^{*}$ is a cocycle that generates $H^{n}\left(\mathbb{R} P^{n} ; \mathbb{Z}_{2}\right)$.

To complete the main goal of this section, we now exhibit an explicit 1-cocycle $u \in C^{1}\left(\mathbb{R} P^{n} ; \mathbb{Z}_{2}\right)$ such that its cohomology class $[u] \in H^{1}\left(\mathbb{R} P^{n} ; \mathbb{Z}_{2}\right)$ satisfies $[u]^{n} \neq 0$. Indeed, define

$$
u:=\sum_{1 \leqslant i<j \leqslant n+1}\left\langle v_{i}, w_{j}\right\rangle^{*} .
$$

Theorem 5.4. The cochain $u$ is a cocycle, i.e. $\delta(u)=0$, and $[u]^{n} \neq 0$.

Proof. To show that $\delta(u)=0$ it suffices to show $0=(\delta(u))(\sigma)=u(d \sigma)$ for each 2simplex $\sigma=\left\langle \pm v_{r}, \pm v_{s}, \pm v_{t}\right\rangle, r<s<t$. Because $\sigma=\tau(\sigma)$, we can suppose without loss of generality that there is at most one minus sign, thus $\sigma$ has one of the four forms $\left\langle v_{r}, v_{s}, v_{t}\right\rangle,\left\langle w_{r}, v_{s}, v_{t}\right\rangle,\left\langle v_{r}, w_{s}, v_{t}\right\rangle,\left\langle v_{r}, v_{s}, w_{t}\right\rangle$. In the first case

$$
d \sigma=\left\langle v_{s}, v_{t}\right\rangle+\left\langle v_{r}, v_{t}\right\rangle+\left\langle v_{r}, v_{s}\right\rangle
$$

and each summand $\left\langle v_{i}, w_{j}\right\rangle^{*}$ of $u$ evaluates to 0 on each of the three terms in $d \sigma$. In the second case

$$
d \sigma=\left\langle v_{s}, v_{t}\right\rangle+\left\langle w_{r}, v_{t}\right\rangle+\left\langle w_{r}, v_{s}\right\rangle=\left\langle v_{s}, v_{t}\right\rangle+\left\langle v_{r}, w_{t}\right\rangle+\left\langle v_{r}, w_{s}\right\rangle
$$

so the summands $\left\langle v_{r}, w_{t}\right\rangle^{*},\left\langle v_{r}, w_{s}\right\rangle^{*}$ of $u$ each evaluate to 1 on $d \sigma$ while all other summands evaluate to 0 , giving $u(d \sigma)=1+1=0$. The third and fourth cases are similar to the second.

Next, for the $n$-fold cup product $[u]^{n}$, one must show that $u^{\otimes n}$ evaluates to 1 on a generator of $H_{n}\left(\mathbb{R} P^{n} ; \mathbb{Z}_{2}\right.$ ). From the above discussion (cf. Lemma 5.1 and the remark just before Theorem 5.2) we can take this generator to be $s_{n}:=\sum_{A \subseteq \underline{n}} \sigma_{A}$ (note that we are using $\mathbb{Z}_{2}$ coefficients). Each $\sigma_{A}$ has $v_{n+1}$ as final vertex, so in the sum $s_{n}$ there will be a single "alternating" $\sigma_{A}$ which we call $\omega$, namely $\omega=$ $\left\langle v_{1}, w_{2}, v_{3}, \ldots, w_{n}, v_{n+1}\right\rangle$ for $n$ even or $\omega=\left\langle w_{1}, v_{2}, w_{3}, \ldots, w_{n}, v_{n+1}\right\rangle$ for $n$ odd. Then $s_{n}=\omega+\varphi$ where $\varphi$ is the sum of all $\sigma_{A}$ with a repetition, i.e. a $v_{i} v_{i+1}$ or $w_{j} w_{j+1}$ in $\sigma_{A}$. 
Now from (3), it is clear that

$$
u^{\otimes n}=\sum_{i_{r}<j_{r}}\left\langle v_{i_{1}}, w_{j_{1}}\right\rangle^{*} \otimes\left\langle v_{i_{2}}, w_{j_{2}}\right\rangle^{*} \otimes \cdots \otimes\left\langle v_{i_{n}}, w_{j_{n}}\right\rangle^{*}
$$

evaluates to zero on all terms of $\varphi$, hence for $n$ even

$$
u^{\otimes n}\left(s_{n}\right)=u^{\otimes n}(\omega)=\left\langle v_{1}, w_{2}\right\rangle^{*}\left(\left\langle v_{1}, w_{2}\right\rangle\right) \cdot\left\langle v_{2}, w_{3}\right\rangle^{*}\left(\left\langle w_{2}, v_{3}\right\rangle\right) \cdots=1^{n}=1,
$$

using that $\left\langle w_{2}, v_{3}\right\rangle=\left\langle v_{2}, w_{3}\right\rangle,\left\langle w_{4}, v_{5}\right\rangle=\left\langle v_{4}, w_{5}\right\rangle$, etc. The argument for $n$ odd is essentially the same. Hence $[u]^{n} \neq 0$.

Corollary 5.5. For each $j, 0 \leqslant j \leqslant n,[u]^{j}$ generates $H^{j}\left(\mathbb{R} P^{n} ; \mathbb{Z}_{2}\right)$, which is thus isomorphic to the truncated polynomial algebra $\mathbb{Z}_{2}[[u]] /\left([u]^{n+1}=0\right)$.

\section{Appendix}

In this appendix we show $H_{j}\left(\mathbb{R} P^{n}, \mathbb{R} P^{n-1}\right)=0,0 \leqslant j \leqslant n-1$, by means of an explicit contracting homotopy $s$ in the chain complex

$$
C_{*}: C_{n} \stackrel{\tilde{d}_{n}}{\longrightarrow} C_{n-1} \stackrel{\tilde{d}_{n-1}}{\longrightarrow} \cdots \stackrel{\tilde{d}_{2}}{\longrightarrow} C_{1} \stackrel{\tilde{d}_{1}}{\longrightarrow} C_{0} \stackrel{\tilde{d}_{0}}{\longrightarrow} 0,
$$

where we write $C_{j}$ for $C_{j}\left(\mathbb{R} P^{n}, \mathbb{R} P^{n-1}\right)$ in this appendix, and as in Section $5 \tilde{d}$ for its differential. Recall that this means a sequence of homomorphisms $s_{j}: C_{j} \rightarrow C_{j+1}$, $0 \leqslant j \leqslant n-1$, with $\tilde{d}_{j+1} s_{j}+s_{j-1} \tilde{d}_{j}=i d_{C_{j}}$ and implies that $H_{j}\left(\mathbb{R} P^{n}, \mathbb{R} P^{n-1}\right)=0$, $0 \leqslant j \leqslant n-1$. Note that $s_{-1}=0$.

Since $C_{*}\left(\mathbb{R} P^{n}, \mathbb{R} P^{n-1}\right)=C_{*}\left(\mathbb{R} P^{n}\right) / C_{*}\left(\mathbb{R} P^{n-1}\right)$, any non-zero simplex $\sigma$ of the chain complex $C_{*}\left(\mathbb{R} P^{n}, \mathbb{R} P^{n-1}\right)$ must contain the vertex $v_{n+1}$. We can therefore write any $r$-simplex $\sigma$ uniquely as

$$
\sigma=\left\langle x_{i_{1}}, x_{i_{2}}, \ldots, x_{i_{r}}, v_{n+1}\right\rangle \text { where } 1 \leqslant i_{1}<i_{2}<\ldots<i_{r} \leqslant n,
$$

and henceforth use this notation. Of course, these are the generators of the free abelian group $C_{*}$.

We next define a homomorphism

$$
\Lambda_{p}: C_{r-p} \rightarrow C_{r}
$$

show it is an anti-chain map (i.e., $\tilde{d} \Lambda_{p}=(-1)^{p} \Lambda_{p} \tilde{d}$ ), prove one combinatorial lemma, and after that it will be fairly easy to construct the contracting homotopy.

Definition 6.1. Let $\sigma=\left\langle x_{m_{1}}, \ldots, x_{m_{r-p}}, v_{n+1}\right\rangle$ be an $(r-p)$-simplex in $C_{r-p}, p+$ $1 \leqslant m_{1}<\ldots<m_{r-p} \leqslant n$. Set

$$
\Lambda_{p}(\sigma)=\sum_{\lambda_{i}= \pm 1}\left(\prod_{i=1}^{p} \lambda_{i}\right)\left\langle\lambda_{1} v_{1}, \ldots, \lambda_{p} v_{p}, \sigma\right\rangle \in C_{r} .
$$

We remark that this sum has $2^{p}$ terms, e.g. $\Lambda_{2}(\sigma)=\left\langle v_{1}, v_{2}, \sigma\right\rangle-\left\langle v_{1}, w_{2}, \sigma\right\rangle-$ $\left\langle w_{1}, v_{2}, \sigma\right\rangle+\left\langle w_{1}, w_{2}, \sigma\right\rangle$.

Lemma 6.2. The map $\Lambda_{p}$ satisfies $\tilde{d} \Lambda_{p}=(-1)^{p} \Lambda_{p} \tilde{d}$. 
Proof. One has

$$
\tilde{d} \Lambda_{p}(\sigma)=\sum_{\lambda_{i}= \pm 1}\left(\prod_{i=1}^{p} \lambda_{i}\right) \tilde{d}\left\langle\lambda_{1} v_{1}, \ldots, \lambda_{p} v_{p}, \sigma\right\rangle .
$$

The first terms of this sum, i.e., those that arise by omitting $\lambda_{1} v_{1}$ (for $\lambda_{1}= \pm 1$ ), gives

$$
\sum_{\lambda_{2}, \ldots, \lambda_{p}= \pm 1}\left(\prod_{i=2}^{p} \lambda_{i}\right)\left(\left\langle\lambda_{2} v_{2}, \ldots, \lambda_{p} v_{p}, \sigma\right\rangle-\left\langle\lambda_{2} v_{2}, \ldots, \lambda_{p} v_{p}, \sigma\right\rangle\right)=0 .
$$

The same happens for the terms omitting $\lambda_{2} v_{2}, \ldots$, omitting $\lambda_{p} v_{p}$. What remains gives us

$$
\tilde{d} \Lambda_{p}(\sigma)=\sum_{\lambda_{i}= \pm 1}\left(\prod_{i=1}^{p} \lambda_{i}\right)\left\langle\lambda_{1} v_{1}, \ldots, \lambda_{p} v_{p},(-1)^{p} \tilde{d} \sigma\right\rangle=(-1)^{p} \Lambda_{p}(\tilde{d} \sigma)
$$

Lemma 6.3. Let $\omega=\left\langle w_{1}, \ldots, w_{p}, \sigma\right\rangle, p \geqslant 1$. Then

$$
\begin{aligned}
& \left\langle v_{1}, w_{2}, \ldots, w_{p}, \sigma\right\rangle-\Lambda_{1}\left\langle v_{2}, w_{3}, \ldots, w_{p}, \sigma\right\rangle+\Lambda_{2}\left\langle v_{3}, w_{4}, \ldots, w_{p}, \sigma\right\rangle-\cdots \\
& \quad+(-1)^{p} \Lambda_{p}\langle\sigma\rangle=\omega .
\end{aligned}
$$

Proof. The final term $(-1)^{p} \Lambda_{p}\langle\sigma\rangle$ clearly has $\omega$ as one of its $2^{p}$ terms (corresponding to $\lambda_{1}=\ldots=\lambda_{p}=-1$ ). It is not difficult to see that the other $2^{p}-1$ terms in $(-1) p \Lambda_{p}\langle\sigma\rangle$ cancel the other $1+2+\cdots+2^{p}=2^{p}-1$ terms in (4) in pairs. Indeed, for each term other than $\omega$ in $(-1)^{p} \Lambda_{p}\langle\sigma\rangle$, we have $1 \leqslant m:=\max \left\{j: \lambda_{j}=-1\right\} \leqslant$ $p$, and one easily sees that this term will cancel the corresponding term in the $(-1)^{m-1} \Lambda_{m}$ term in (4), (where $\Lambda_{0}$ is the first term).

Now to define a contracting homotopy $s$, we start with $s_{0}\left(v_{n+1}\right)=\left\langle v_{1}, v_{n+1}\right\rangle$, then $\tilde{d} s_{0}\left(v_{n+1}\right)=\left\langle v_{n+1}\right\rangle$ as required.

Definition 6.4. For $0<r<n$, we define $s_{r}\left\langle x_{i_{1}}, \ldots, x_{i_{r}}, v_{n+1}\right\rangle=s_{r}(\omega)$ using the following four cases.

1. If $i_{1}>1$, then $s_{r}(\omega)=\left\langle v_{1}, \omega\right\rangle$,

2. If $i_{1}=1$ and $x_{i_{1}}=v_{1}$, then $s_{r}(\omega)=0$,

3. If $\omega=\left\langle w_{1}, \ldots, w_{p}, x_{i_{p+1}}, \ldots, v_{n+1}\right\rangle=\left\langle w_{1}, \ldots, w_{p}, \sigma\right\rangle$ with $p<i_{p+1}$, then $s_{r}(\omega)=$ $\Lambda_{p}\left(\left\langle v_{p+1}, \sigma\right\rangle\right)$,

4. (remaining cases) If $\omega=\left\langle w_{1}, \ldots, w_{p}, v_{p+1}, x_{i_{p+2}}, \ldots, v_{n+1}\right\rangle$, then $s_{r}(\omega)=0$.

Proposition 6.5. The homomorphism $s$ is a contracting homotopy for $C_{*}$.

Proof. We prove that $s$ is a contracting homotopy for the cases 1 to 4 in the given order. In fact, 1,2 and 4 are routine verifications and we give only the proof of 3 here. 
Let $\omega=\left\langle w_{1}, w_{2}, \ldots, w_{p}, \sigma\right\rangle$, where $p \geqslant 1$.

$$
\begin{aligned}
\tilde{d} s_{r}(\omega)+s_{r-1} \tilde{d}(\omega)= & \tilde{d} \Lambda_{p}\left(\left\langle v_{p+1}, \sigma\right\rangle\right)+s_{r-1}\left(\left\langle w_{2}, \ldots, w_{p}, \sigma\right\rangle-\left\langle w_{1}, w_{3}, \ldots, w_{p}, \sigma\right\rangle\right. \\
& \left.+\left\langle w_{1}, w_{2}, w_{4}, \ldots, w_{p}, \sigma\right\rangle-\cdots+(-1)^{p}\left\langle w_{1}, \ldots, w_{p}, \tilde{d} \sigma\right\rangle\right) .
\end{aligned}
$$

Applying Lemma 6.2, we get

$$
\begin{aligned}
\tilde{d} s_{r}(\omega)+s_{r-1} \tilde{d}(\omega)= & (-1)^{p} \Lambda_{p} \tilde{d}\left\langle v_{p+1}, \sigma\right\rangle+\left\langle v_{1}, w_{2}, \ldots, w_{p}, \sigma\right\rangle-\Lambda_{1}\left\langle v_{2}, w_{3}, \ldots, w_{p}, \sigma\right\rangle \\
& +\Lambda_{2}\left\langle v_{3}, w_{4}, \ldots, w_{p}, \sigma\right\rangle-\cdots+(-1)^{p} \Lambda_{p}\left\langle v_{p+1}, \tilde{d} \sigma\right\rangle \\
= & (-1)^{p} \Lambda_{p}\langle\sigma\rangle-(-1)^{p} \Lambda_{p}\left\langle v_{p+1}, \tilde{d} \sigma\right\rangle+\left\langle v_{1}, w_{2}, \ldots, w_{p}, \sigma\right\rangle \\
& -\Lambda_{1}\left\langle v_{2}, w_{3}, \ldots, w_{p}, \sigma\right\rangle+\Lambda_{2}\left\langle v_{3}, w_{4}, \ldots, w_{p}, \sigma\right\rangle-\ldots \\
& +(-1)^{p} \Lambda_{p}\left\langle v_{p+1}, \tilde{d} \sigma\right\rangle .
\end{aligned}
$$

Canceling the second and final terms, Lemma 6.3 shows that the remaining terms equal $\omega$.

\section{Acknowledgments}

The authors are grateful to the referee for suggestions which led to substantial improvements in the original version of this paper.

\section{References}

[1] B. Basak and S. Sarkar, Equilibrium and equivariant triangulations of some small covers with minimum number of vertices, J. Ramanujan Math. Soc. 30, no. 1 (2015), 29-50.

[2] W.F. Basener, Topology and Its Applications, John Wiley \& Sons Inc., Hoboken (2006).

[3] H.S.M. Coxeter, Regular Polytopes, Third edition, Dover Publications, Inc., New York, 1973.

[4] E.B. Curtis, Simplicial homotopy theory, Adv. in Math. 6 (1971), 107-209.

[5] B. Datta, Minimal triangulations of manifolds, J. Indian Inst. Sci. 87, no. 4 (2007), 429-449.

[6] J. Dieudonné, A History of Algebraic and Differential Topology, 1900-1960. Birkhäuser Boston, Inc., Boston, 1989.

[7] S. Eilenberg and J.A. Zilber, Semi-simplicial complexes and singular homology, Ann. of Math. 51, no. 3 (1950), 499-513.

[8] J.B. Giever, On the equivalence of two singular homology theories, Ann. of Math. (2) 51 (1950), 178-191.

[9] S. Galatius and O. Randal-Williams, Stable moduli spaces of high-dimensional manifolds, Acta Math. 212, no. 2 (2014), 257-377.

[10] P.G. Goerss and J.F. Jardine, Simplicial Homotopy Theory, Progr. Math., 174. Birkhäuser Verlag, Basel, 1999.

[11] A. Hatcher, Algebraic Topology, Cambridge Univ. Press, Cambridge, 2002, First edition. https://www.math.cornell.edu/ hatcher/AT/AT.pdf, Third edition. 
[12] P. Hilton and S. Wylie, Homology Theory, an Introduction to Algebraic Topology, Cambridge Univ. Press, Cambridge, 1960.

[13] S.T. Hu, On the realizability of homotopy groups and their operations, Pacific J. Math. 1 (1951), 583-602.

[14] S. Illman, Smooth equivariant triangulations of G-manifolds, Math. Ann. 233 (1978), 199-220.

[15] D.M. Kan, On c. s. s. complexes, Amer. J. Math. 79 (1957), 449-476.

[16] S. MacLane, Categories for the Working Mathematician, Grad. Texts Math., 5. Heidelberg, Berlin, 1971.

[17] J.P. May, Simplicial Objects in Algebraic Topology, Chicago Lectures Math. Ser., Univ. of Chicago Press, Chicago, 1967.

[18] J.E. McClure, On semisimplicial sets satisfying the Kan condition, Homology Homotopy Appl. 15, no. 1 (2013), 73-82.

[19] J. Milnor, The geometric realization of a semi-simplicial complex, Ann. of Math. (2) 65 (1957), 357-362.

[20] J.C. Moore, Semi-simplicial complexes and Postnikov systems. 1958 Symposium internacional de topología algebraica International symposium on algebraic topology, pp. 232-247 Universidad Nacional Autónoma de México and UNESCO, Mexico City.

[21] C.P. Rourke and B.J. Sanderson, $\Delta$-sets. I. Homotopy theory, Quart. J. Math. Oxford Ser. (2) 22 (1971), 321-338.

[22] P. Selick, Introduction to Homotopy Theory, Fields Inst. Monogr., 9. Amer. Math. Soc., Providence, 1997.

[23] C.A. Weibel, History of homological algebra, History of Topology, pp. 797-836, North-Holland, Amsterdam, 1999.

Michael Pors mike.pors@gmail.com

Department of Mathematics and Statistics, University of Calgary, Calgary, Alberta, Canada T2N 1N4

Soumen Sarkar soumensarkar20@gmail.com

Department of Mathematics and Statistics, University of Calgary, Calgary, Alberta, Canada T2N 1N4

Peter Zvengrowski zvengrow@ucalgary.ca

Department of Mathematics and Statistics, University of Calgary, Calgary, Alberta, Canada T2N $1 \mathrm{~N} 4$ 